\title{
Effect of Dates of Sowing on Incidence of Insect Pests on Castor Genotypes
}

\author{
G. Laxman* and T. Uma Maheswari \\ Department of Entomology, College of Agriculture, Rajendranagar, \\ Hyderabad-500030, Telangana, India \\ *Corresponding author
}

\begin{tabular}{|c|c|}
\hline & A B S T R A C T \\
\hline & Study on effect of dates of sowing on incidence of insect pests on castor \\
\hline $\begin{array}{l}\text { Ke e y w o r d s } \\
\text { Effects, Castor, } \\
\text { Achaea janata, } \\
\text { Spodoptera litura. }\end{array}$ & $\begin{array}{l}\text { genotypes revealed that incidence of Achaea janata was observed when } \\
\text { castor was sown either early or late, however there was a decrease in larval } \\
\text { incidence when the crop was sown late during the month of August as } \\
\text { compared to that during July. Maximum of } 2.73 \text { larvae were observed on }\end{array}$ \\
\hline Article Info & $\begin{array}{l}48-1 \text { when sown early which was reduced to } 1.07 \text { with late sowing. } \\
\text { Incidence of } S \text {. litura was high in early sown crop compared to late sown }\end{array}$ \\
\hline $\begin{array}{l}\text { Accepted: } \\
\text { 12 September } 2017 \\
\text { Available Online: } \\
\text { 10 November } 2017\end{array}$ & $\begin{array}{l}\text { crop. In case of early sown castor, high larval population was recorded on } \\
48-1 \text { (3.20) and no larvae were observed on DPC-9 and in case of late sown } \\
\text { crop high larval population was observed on DCS-9 (1.40) where no larvae } \\
\text { were observed on DPC-9. }\end{array}$ \\
\hline
\end{tabular}

\section{Introduction}

Castor (Ricinus communis Lin.) in an important non edible oilseed crop which is grown in arid and semi-arid regions. In India, Castor is sown during July or August and harvested around December or January. More than 20 species of insect pests were found associated with castor, but many of them were highly irregular in occurrence over years, distributed in patches with low population causing no remarkable damage to the crop. Only 10 species belonging to Lepidoptera, Hemiptera, Orthoptera and Thysanoptera showed variable economic importance and of them, five species were found regular with high degree of severity as major pests. Most of the insect pests were either defoliators or sucking pests (Sarma et al., 2005). The magnitude of insect pest problem is quite high in Southern India where castor is grown mainly as rainfed crop, resulting in lower seed yield. The major pest problems in castor include the defoliators, semilooper, Achaea janata L., tobacco caterpillar, Spodoptera litura Fab. capsule borer, Conogethes punctiferalis Guen. and the sucking pests, leafhopper, Empoasca flavescens Fab. thrips, Retithrips syniacus Mayet and whitefly, Trialeurodes ricini Misra (DOR, 2005; Lakshminarayana and Raoof, 2005). Studies on relationship between different dates of sowing aid to find out the incidence of insect pests which helps to forewarn the cultivators 
to resort to preventive measures against such pests in time. Usually, pest occurrence and its population fluctuations depend on the changing environmental situation.

\section{Materials and Methods}

The field was ploughed thoroughly thrice to obtain fine tilth and propely levelled after removing stubbles, weeds and trash. Two sowings were taken up on 30.07.2013 and 30.08 .2013 by dibling the seed with a spacing of $90 \times 60 \mathrm{cms}$ at the rate of two seeds per hill. Gap filling was done a week after germination. The seedlings were thinned out within 15 days after sowing allowing one healthy seedling to grow for hill. The recommended dose of fertilizer i.e., $80 \mathrm{~kg} \mathrm{~N}$, $60 \mathrm{~kg} \mathrm{P}$ and $40 \mathrm{~kg} \mathrm{~K} / \mathrm{ha}$ was applied, of which half dose of nitrogen and the entire dose of potash and phosphorus were applied as basal and rest of nitrogen at 30, 60 and 90 days after germination in equal doses. The experimental plots were irrigated as and when required. Pre-emergence herbicide Pendimethalin 30 EC was applied a day after sowing to arrest the growth of weeds. Hand weeding was done twice so as to keep the crop free of weeds. Observations recorded on larvae per plant at 30, 60, 90, 120 and 150 days of crop for castor semilooper and tobacco caterpillar.

\section{Results and Discussion}

Effect of early sowing on incidence of castor semilooper, Achaea janata

Studies were conducted on effect of early and late sowing of castor genotypes, with an interval of 30 days during month of July and August, 2013 and the data recorded on incidence of defoliators i.e. A. janata and $S$. litura during these two periods of crop growth are presented in the table 1. It was clear from the present study that incidence of A. janata though started from 30 days after sowing but comparatively significant population was recorded from 60 days after sowing. Among the genotypes, maximum of 4.67 larvae was observed on RG-2928 followed by 3.67 in $\mathrm{PCH}-106$ and 3.0 in GCH-4. However, in other genotypes, lowest of 1 larva and highest of 1.67 larvae and no larvae were observed on DCS-9. At 90 days after sowing maximum of 3.33 larvae were observed on RG-2928, where majority of the genotypes have recorded an average of 1.00 to 1.67 larvae per plant. By 120 days crop age, the larval population remained almost same or with a slight decrease or increase from the same at 90 days. However, by 150 days after sowing, absolutely no larvae were recorded feeding on PCH-248 whereas on other genotypes, the larval population ranged between 0.67-1.33 in DPC-9. On an average, when the genotypes were sown early during the month of July, among all genotypes, maximum of 2.73 larvae of $A$. janata were observed on 48-1 followed by 2.04 larvae in RG-2928, 2.02 larvae in Kranthi, 2.00 larvae in $\mathrm{GCH}-4$ and PCH-106 where other genotypes recorded minimum of one larva per plant.

\section{Effect of late sowing on incidence of castor semilooper, Achaea janata}

When sowing of castor was delayed by 30 days, no significant effect incidence of $A$. janata was observed up to 60 days after sowing. After 90 days, larval population to a maximum of 2.67 was recorded on DCS-9, PCH-222 and RG-1180 followed by 2.33 larvae on 48-1, RG-2835, PCH-106 and 2.00 larvae on Kranthi. Very low population of $A$. janata i.e. one larvae per plant was observed on DPC-9, Haritha, PCH-288, PCH-111, PCH-254, M-574, PCH-248, PCH-294, GCH4, PCH-282 and 1.33 in RG-776 and 1.67 on $\mathrm{PCH}-262$. As the age of the crop increased to 120 days, total larval population per plant was reduced on all genotypes. However, highest 
of 0.33 followed by 0.67 larvae i.e. less than 1.00 larva was observed on all genotypes and zero population on DPC-9, GCH-4 and RG776. By 150 days, no pest incidence was observed on any of the genotypes (Table 2).

\section{Effect of early sowing on incidence of tobacco caterpillar, Spodoptera litura}

Studies were conducted on influence of different dates of sowing of castor genotypes during month of July and August, 2013 with 30 days interval and the results are presented in the table 3. It is evident from the study that when castor is sown early i.e. during July and data was recorded 30 days after sowing, incidence of $S$. litura was observed throughout the crop growth period. At 30 days after sowing, majority of the genotypes have recorded either one or two larvae except the genotypes viz., DPC-9, PCH-248, GCH-4, PCH-222 and RG-2928 on which not even a single larva was observed. However, at 60 days after sowing, all the genotypes have showed larval population ranging from 1.00 to 3.00 except DPC-9 which did not record a single larva of S. litura. At 90 days age crop, significantly maximum of 6.00 larvae per plant was observed in PCH-262 followed by 48-1, RG-1180 and Kranthi with five larvae per plant which remained on par with each other. Four larvae per plant were recorded in the case of Haritha, PCH-111, RG-2835, PCH-222 and RG-776. Minimum of one larva was observed in the genotypes, PCH-288, M574 and $\mathrm{PCH}-248$. Absolutely even at 90 days after sowing no larval population was observed in the case of genotypes viz., DPC-9 and RG-2928. At 120 days age of the crop, maximum of 4.00 larvae were recorded on DCS-9, 48-1 and PCH-288 and 3.00 larvae on PCH-294 and RG-1180. Majority of the genotypes have recorded only 2.00 larvae per plant as seen in the case of Haritha, PCH-111, PCH-248, GCH-4, RG-2835, RG-2928, RG776 and Kranthi. Minimum of 1.00 larva was seen in the case of PCH-262, PCH-282, PCH106 and PCH-222 where no S. litura infestation was observed on DPC-9. At 150 days after sowing, maximum of only one larva was observed on the genotypes where no larva was observed in the genotypes, DPC9, Haritha, PCH-288, PCH-254, Kiran, $\mathrm{PCH}-$ 294, PCH-262, PCH-282, PCH-106, PCH222, RG-2928 and RG-776. On an average, no $S$. litura incidence was observed on DPC-9 throughout the crop growth period and maximum of 3.2 larvae on 48-1 was recorded. However, mean number of larvae of 2.4 was observed on RG-2835, RG-1180 and Kranthi followed by 2.2 larvae on DCS-9 and PCH262 and 2.0 larvae in the case of PCH-111. Larval population ranging from 1.0 to 1.8 larvae per plant was observed in the genotypes, Haritha (1.80), PCH-288 (1.20), kiran (1.20), PCH-294 (1.60), GCH-4 (1.20), PCH-282 (1.20), PCH-106 (1.40), PCH-222 (1.40) and RG-776 (1.60). Minimum of 1.0 larva per plant was recorded in the genotypes, PCH-254, M-574, PCH-248 and RG-1180.

\section{Effect of late sowing on incidence of tobacco caterpillar, Spodoptera litura}

When sowing of castor genotypes delayed by 30 days i.e. sowing was done during month of August, data was recorded from 30 days onwards and presented in the table 4 which reveals that all the genotypes significantly differed in incidence of S. litura at 30 days after sowing where majority of the genotypes have recorded either one or two larvae except on the genotypes viz., DPC-9, PCH-111, Kiran, PCH-294, PCH-262, RG-2928 and RG-1180 on which not even a single larva was observed. However, at 60 days after sowing, all the genotypes have showed larval population ranging from 1.00 to 3.00 except DPC-9, 48-1, PCH-111PCH-248, PCH-262, RG-2835, PCH-106, RG-2928, RG-776 and Kranthi on which no infestation of $S$. litura was recorded. 
Table.1 Effect of early sowing on incidence of castor semilooper, Achaea janata

\begin{tabular}{|c|c|c|c|c|c|c|}
\hline \multirow[b]{2}{*}{ Genotypes } & \multicolumn{6}{|c|}{ Number of larvae/plant } \\
\hline & $\begin{array}{c}30 \mathrm{das} \\
(30.09 .2013)\end{array}$ & $\begin{array}{c}60 \mathrm{das} \\
(30.10 .2013)\end{array}$ & $\begin{array}{c}90 \text { das } \\
(30.11 .2013)\end{array}$ & $\begin{array}{c}120 \text { das } \\
(30.12 .2013)\end{array}$ & $\begin{array}{c}150 \text { das } \\
(30.01 .2014)\end{array}$ & Mean \\
\hline DPC-9 & $\begin{array}{c}0.67 \\
(1.28) \\
\end{array}$ & $\begin{array}{c}1.67 \\
(1.63) \\
\end{array}$ & $\begin{array}{c}1.00 \\
(1.38) \\
\end{array}$ & $\begin{array}{c}1.00 \\
(1.41) \\
\end{array}$ & $\begin{array}{c}1.33 \\
(1.52) \\
\end{array}$ & $\begin{array}{c}1.13 \\
(1.46) \\
\end{array}$ \\
\hline DCS-9 & $\begin{array}{c}0.00 \\
(1.00)\end{array}$ & $\begin{array}{c}0.33 \\
(1.34)\end{array}$ & $\begin{array}{c}0.00 \\
(1.00)\end{array}$ & $\begin{array}{c}0.00 \\
(1.00)\end{array}$ & $\begin{array}{c}0.67 \\
(1.28)\end{array}$ & $\begin{array}{c}0.20 \\
(1.17)\end{array}$ \\
\hline $48-1$ & $\begin{array}{c}1.00 \\
(1.38) \\
\end{array}$ & $\begin{array}{c}1.33 \\
(1.52) \\
\end{array}$ & $\begin{array}{c}0.67 \\
(1.24) \\
\end{array}$ & $\begin{array}{l}7.33 \\
(2.9) \\
\end{array}$ & $\begin{array}{c}3.33 \\
(2.08) \\
\end{array}$ & $\begin{array}{c}2.73 \\
(1.84) \\
\end{array}$ \\
\hline Haritha & $\begin{array}{c}0.67 \\
(1.28) \\
\end{array}$ & $\begin{array}{c}1.67 \\
(1.58)\end{array}$ & $\begin{array}{c}1.67 \\
(1.63)\end{array}$ & $\begin{array}{c}2.00 \\
(1.72)\end{array}$ & $\begin{array}{c}1.67 \\
(1.61)\end{array}$ & $\begin{array}{l}1.54 \\
(1.6) \\
\end{array}$ \\
\hline РCH-288 & $\begin{array}{c}1.33 \\
(1.52) \\
\end{array}$ & $\begin{array}{c}1.33 \\
(1.52) \\
\end{array}$ & $\begin{array}{c}1.33 \\
(1.49) \\
\end{array}$ & $\begin{array}{c}0.67 \\
(1.28) \\
\end{array}$ & $\begin{array}{c}0.67 \\
(1.24) \\
\end{array}$ & $\begin{array}{c}1.07 \\
(1.43) \\
\end{array}$ \\
\hline PCH-111 & $\begin{array}{c}1.00 \\
(1.38)\end{array}$ & $\begin{array}{c}1.33 \\
(1.52)\end{array}$ & $\begin{array}{c}1.33 \\
(1.52)\end{array}$ & $\begin{array}{c}1.33 \\
(1.59)\end{array}$ & $\begin{array}{c}1.00 \\
(1.38)\end{array}$ & $\begin{array}{l}1.20 \\
(1.5) \\
\end{array}$ \\
\hline PCH-254 & $\begin{array}{c}0.33 \\
(1.34)\end{array}$ & $\begin{array}{c}1.00 \\
(1.41)\end{array}$ & $\begin{array}{c}1.00 \\
(1.38)\end{array}$ & $\begin{array}{c}1.00 \\
(1.41)\end{array}$ & $\begin{array}{c}1.33 \\
(1.48)\end{array}$ & $\begin{array}{l}0.93 \\
(1.4)\end{array}$ \\
\hline M-574 & $\begin{array}{c}0.33 \\
(1.34)\end{array}$ & $\begin{array}{c}1.00 \\
(1.41)\end{array}$ & $\begin{array}{c}1.00 \\
(1.41)\end{array}$ & $\begin{array}{c}2.00 \\
(1.72)\end{array}$ & $\begin{array}{c}1.67 \\
(1.62)\end{array}$ & $\begin{array}{l}1.20 \\
(1.5)\end{array}$ \\
\hline Kiran & $\begin{array}{c}0.67 \\
(1.28)\end{array}$ & $\begin{array}{c}1.67 \\
(1.63)\end{array}$ & $\begin{array}{c}2.00 \\
(1.73)\end{array}$ & $\begin{array}{c}1.00 \\
(1.41)\end{array}$ & $\begin{array}{c}1.00 \\
(1.38)\end{array}$ & $\begin{array}{c}1.27 \\
(1.49)\end{array}$ \\
\hline PCH-248 & $\begin{array}{c}0.33 \\
(1.34) \\
\end{array}$ & $\begin{array}{c}1.00 \\
(1.33) \\
\end{array}$ & $\begin{array}{c}1.00 \\
(1.41) \\
\end{array}$ & $\begin{array}{c}0.33 \\
(1.14) \\
\end{array}$ & $\begin{array}{c}0.00 \\
(1.00) \\
\end{array}$ & $\begin{array}{c}0.53 \\
(1.22) \\
\end{array}$ \\
\hline PCH-294 & $\begin{array}{c}0.67 \\
(1.28)\end{array}$ & $\begin{array}{c}1.00 \\
(1.41)\end{array}$ & $\begin{array}{c}1.00 \\
(1.41)\end{array}$ & $\begin{array}{c}1.33 \\
(1.52)\end{array}$ & $\begin{array}{c}1.33 \\
(1.48)\end{array}$ & $\begin{array}{c}1.07 \\
(1.44) \\
\end{array}$ \\
\hline PCH-262 & $\begin{array}{c}0.67 \\
(1.28)\end{array}$ & $\begin{array}{c}1.33 \\
(1.52)\end{array}$ & $\begin{array}{c}0.33 \\
(1.14)\end{array}$ & $\begin{array}{c}0.67 \\
(1.24)\end{array}$ & $\begin{array}{c}0.67 \\
(1.24)\end{array}$ & $\begin{array}{c}0.73 \\
(1.31)\end{array}$ \\
\hline GCH-4 & $\begin{array}{c}0.33 \\
(1.34)\end{array}$ & $\begin{array}{c}3.00 \\
(1.99)\end{array}$ & $\begin{array}{c}1.67 \\
(1.61)\end{array}$ & $\begin{array}{c}2.33 \\
(1.82)\end{array}$ & $\begin{array}{c}2.67 \\
(1.90)\end{array}$ & $\begin{array}{c}2.00 \\
(1.71) \\
\end{array}$ \\
\hline РCH-282 & $\begin{array}{c}0.33 \\
(1.34)\end{array}$ & $\begin{array}{c}1.00 \\
(1.41)\end{array}$ & $\begin{array}{c}1.00 \\
(1.41)\end{array}$ & $\begin{array}{c}1.00 \\
(1.38)\end{array}$ & $\begin{array}{c}1.33 \\
(1.52)\end{array}$ & $\begin{array}{l}0.93 \\
(1.4)\end{array}$ \\
\hline RG-2835 & $\begin{array}{c}1.00 \\
(1.41)\end{array}$ & $\begin{array}{c}1.67 \\
(1.63)\end{array}$ & $\begin{array}{c}1.67 \\
(1.61)\end{array}$ & $\begin{array}{c}1.67 \\
(1.62)\end{array}$ & $\begin{array}{c}1.00 \\
(1.41)\end{array}$ & $\begin{array}{l}1.40 \\
(1.55)\end{array}$ \\
\hline PCH-106 & $\begin{array}{c}1.33 \\
(1.52)\end{array}$ & $\begin{array}{c}3.67 \\
(2.15)\end{array}$ & $\begin{array}{c}2.00 \\
(1.73)\end{array}$ & $\begin{array}{c}1.33 \\
(1.52)\end{array}$ & $\begin{array}{c}1.67 \\
(1.62)\end{array}$ & $\begin{array}{c}2.00 \\
(1.72)\end{array}$ \\
\hline PCH-222 & $\begin{array}{c}1.33 \\
(1.52) \\
\end{array}$ & $\begin{array}{c}1.33 \\
(1.52) \\
\end{array}$ & $\begin{array}{c}1.33 \\
(1.48) \\
\end{array}$ & $\begin{array}{c}3.00 \\
(2.00) \\
\end{array}$ & $\begin{array}{c}1.00 \\
(1.41)\end{array}$ & $\begin{array}{l}1.60 \\
(1.6) \\
\end{array}$ \\
\hline RG-2928 & $\begin{array}{c}0.67 \\
(1.28) \\
\end{array}$ & $\begin{array}{c}4.67 \\
(2.39) \\
\end{array}$ & $\begin{array}{c}3.33 \\
(2.06) \\
\end{array}$ & $\begin{array}{c}1.00 \\
(1.38) \\
\end{array}$ & $\begin{array}{c}0.67 \\
(1.28) \\
\end{array}$ & $\begin{array}{l}2.07 \\
(1.7) \\
\end{array}$ \\
\hline RG-1180 & $\begin{array}{c}1.67 \\
(7.42)\end{array}$ & $\begin{array}{c}1.00 \\
(1.41)\end{array}$ & $\begin{array}{c}1.00 \\
(1.38)\end{array}$ & $\begin{array}{c}2.67 \\
(1.90)\end{array}$ & $\begin{array}{c}2.00 \\
(1.72)\end{array}$ & $\begin{array}{c}1.67 \\
(1.62) \\
\end{array}$ \\
\hline RG-776 & $\begin{array}{c}0.67 \\
(1.28)\end{array}$ & $\begin{array}{c}0.67 \\
(1.24)\end{array}$ & $\begin{array}{c}1.00 \\
(1.41)\end{array}$ & $\begin{array}{c}1.67 \\
(1.61)\end{array}$ & $\begin{array}{c}2.00 \\
(1.67)\end{array}$ & $\begin{array}{l}1.20 \\
(1.5)\end{array}$ \\
\hline Kranthi & $\begin{array}{c}1.00 \\
(1.28) \\
\end{array}$ & $\begin{array}{c}2.00 \\
(1.71) \\
\end{array}$ & $\begin{array}{c}2.67 \\
(1.90) \\
\end{array}$ & $\begin{array}{c}3.00 \\
(2.00) \\
\end{array}$ & $\begin{array}{c}1.67 \\
(1.58) \\
\end{array}$ & $\begin{array}{c}2.02 \\
(1.75) \\
\end{array}$ \\
\hline SEm & & 0.12 & 0.14 & 0.14 & 0.17 & 0.10 \\
\hline $\begin{array}{l}\mathrm{CD} \\
(\mathrm{P}=0.05)\end{array}$ & NS & 0.36 & 0.39 & 0.41 & 0.50 & 0.29 \\
\hline
\end{tabular}

Figures in parenthesis are square root transformed values.

$\mathrm{SEm}=$ Standard error of mean

$\mathrm{CD}(\mathrm{P}=0.05)=$ Critical Difference at $5 \%$ level of significance

DAS $=$ Days after sowing. NS=Non-significant 
Table.2 Effect of late sowing on incidence of castor semilooper, Achaea janata

\begin{tabular}{|c|c|c|c|c|c|c|}
\hline \multirow[b]{2}{*}{ Genotypes } & \multicolumn{6}{|c|}{ Number of larvae/plant } \\
\hline & $\begin{array}{c}30 \text { das } \\
(30.09 .2013)\end{array}$ & $\begin{array}{c}60 \text { das } \\
(30.10 .2013)\end{array}$ & $\begin{array}{c}90 \text { das } \\
(30.11 .2013)\end{array}$ & $\begin{array}{c}120 \text { das } \\
(30.12 .2013)\end{array}$ & $\begin{array}{c}150 \text { das } \\
(30.01 .2014)\end{array}$ & Mean \\
\hline DPC-9 & $\begin{array}{c}1.00 \\
(1.41) \\
\end{array}$ & $\begin{array}{c}0.67 \\
(1.24) \\
\end{array}$ & $\begin{array}{c}1.00 \\
(1.38) \\
\end{array}$ & $\begin{array}{c}0.00 \\
(1.00) \\
\end{array}$ & $\begin{array}{c}0.00 \\
(1.00) \\
\end{array}$ & $\begin{array}{c}0.53 \\
(1.46) \\
\end{array}$ \\
\hline DCS-9 & $\begin{array}{c}0.33 \\
(1.14)\end{array}$ & $\begin{array}{c}1.00 \\
(1.38)\end{array}$ & $\begin{array}{c}2.67 \\
(1.90)\end{array}$ & $\begin{array}{c}1.00 \\
(1.38)\end{array}$ & $\begin{array}{c}0.00 \\
(1.00)\end{array}$ & $\begin{array}{l}1.00 \\
(1.3)\end{array}$ \\
\hline $48-1$ & $\begin{array}{c}1.00 \\
(1.38)\end{array}$ & $\begin{array}{c}0.33 \\
(1.34) \\
\end{array}$ & $\begin{array}{c}2.33 \\
(1.82) \\
\end{array}$ & $\begin{array}{c}1.67 \\
(1.63) \\
\end{array}$ & $\begin{array}{c}0.00 \\
(1.00)\end{array}$ & $\begin{array}{c}1.07 \\
(1.34) \\
\end{array}$ \\
\hline Haritha & $\begin{array}{c}1.33 \\
(1.52)\end{array}$ & $\begin{array}{c}1.67 \\
(1.61)\end{array}$ & $\begin{array}{c}1.00 \\
(1.41)\end{array}$ & $\begin{array}{c}0.67 \\
(1.24)\end{array}$ & $\begin{array}{c}0.00 \\
(1.00)\end{array}$ & $\begin{array}{c}0.93 \\
(1.52)\end{array}$ \\
\hline PCH-288 & $\begin{array}{c}1.00 \\
(1.41)\end{array}$ & $\begin{array}{c}1.33 \\
(1.52)\end{array}$ & $\begin{array}{c}1.00 \\
(1.41)\end{array}$ & $\begin{array}{c}0.33 \\
(1.14)\end{array}$ & $\begin{array}{c}0.00 \\
(1.00)\end{array}$ & $\begin{array}{c}0.93 \\
(1.43) \\
\end{array}$ \\
\hline PCH-111 & $\begin{array}{c}0.67 \\
(1.24)\end{array}$ & $\begin{array}{c}0.67 \\
(1.28)\end{array}$ & $\begin{array}{c}1.00 \\
(1.38)\end{array}$ & $\begin{array}{c}0.33 \\
(1.14)\end{array}$ & $\begin{array}{c}0.00 \\
(1.00)\end{array}$ & $\begin{array}{l}0.53 \\
(1.5)\end{array}$ \\
\hline PCH-254 & $\begin{array}{c}0.33 \\
(1.34)\end{array}$ & $\begin{array}{c}0.67 \\
(1.28)\end{array}$ & $\begin{array}{c}1.00 \\
(1.41)\end{array}$ & $\begin{array}{c}0.67 \\
(1.28)\end{array}$ & $\begin{array}{c}0.00 \\
(1.00)\end{array}$ & $\begin{array}{l}0.53 \\
(1.4)\end{array}$ \\
\hline M-574 & $\begin{array}{c}0.67 \\
(1.24)\end{array}$ & $\begin{array}{c}1.00 \\
(1.41)\end{array}$ & $\begin{array}{c}1.00 \\
(1.41)\end{array}$ & $\begin{array}{c}0.67 \\
(1.28)\end{array}$ & $\begin{array}{c}0.00 \\
(1.00)\end{array}$ & $\begin{array}{l}0.66 \\
(1.5) \\
\end{array}$ \\
\hline Kiran & $\begin{array}{c}0.67 \\
(1.24)\end{array}$ & $\begin{array}{c}1.00 \\
(1.41)\end{array}$ & $\begin{array}{c}0.67 \\
(1.28)\end{array}$ & $\begin{array}{c}0.00 \\
(1.00)\end{array}$ & $\begin{array}{c}0.00 \\
(1.00)\end{array}$ & $\begin{array}{l}0.49 \\
(1.5)\end{array}$ \\
\hline PCH-248 & $\begin{array}{c}1.00 \\
(1.41)\end{array}$ & $\begin{array}{c}0.67 \\
(1.24)\end{array}$ & $\begin{array}{c}1.00 \\
(1.41)\end{array}$ & $\begin{array}{c}0.33 \\
(1.34)\end{array}$ & $\begin{array}{c}0.00 \\
(1.00)\end{array}$ & $\begin{array}{c}0.80 \\
(1.23) \\
\end{array}$ \\
\hline PCH-294 & $\begin{array}{c}0.67 \\
(1.24) \\
\end{array}$ & $\begin{array}{c}0.67 \\
(1.24) \\
\end{array}$ & $\begin{array}{c}1.00 \\
(1.38) \\
\end{array}$ & $\begin{array}{c}1.00 \\
(1.41) \\
\end{array}$ & $\begin{array}{c}0.00 \\
(1.00) \\
\end{array}$ & $\begin{array}{r}0.67 \\
(144) \\
\end{array}$ \\
\hline PCH-262 & $\begin{array}{c}0.67 \\
(1.24) \\
\end{array}$ & $\begin{array}{c}0.67 \\
(1.28) \\
\end{array}$ & $\begin{array}{c}1.67 \\
(1.62) \\
\end{array}$ & $\begin{array}{c}1.00 \\
(1.41) \\
\end{array}$ & $\begin{array}{c}0.00 \\
(1.00) \\
\end{array}$ & $\begin{array}{r}0.60 \\
(1.31) \\
\end{array}$ \\
\hline GCH-4 & $\begin{array}{c}0.67 \\
(1.24)\end{array}$ & $\begin{array}{c}1.00 \\
(1.41)\end{array}$ & $\begin{array}{c}1.00 \\
(1.41)\end{array}$ & $\begin{array}{c}0.00 \\
(1.00)\end{array}$ & $\begin{array}{c}0.00 \\
(1.00)\end{array}$ & $\begin{array}{c}0.53 \\
(1.55)\end{array}$ \\
\hline PCH-282 & $\begin{array}{c}0.67 \\
(1.24)\end{array}$ & $\begin{array}{c}1.00 \\
(1.38)\end{array}$ & $\begin{array}{c}1.00 \\
(1.41)\end{array}$ & $\begin{array}{c}1.67 \\
(1.63)\end{array}$ & $\begin{array}{c}0.00 \\
(1.00)\end{array}$ & $\begin{array}{l}0.90 \\
(1.4)\end{array}$ \\
\hline RG-2835 & $\begin{array}{c}1.33 \\
(1.52) \\
\end{array}$ & $\begin{array}{c}1.67 \\
(1.61) \\
\end{array}$ & $\begin{array}{c}2.33 \\
(1.82) \\
\end{array}$ & $\begin{array}{c}1.00 \\
(1.38) \\
\end{array}$ & $\begin{array}{c}0.00 \\
(1.00) \\
\end{array}$ & $\begin{array}{c}1.27 \\
(1.48) \\
\end{array}$ \\
\hline PCH-106 & $\begin{array}{c}2.00 \\
(1.72)\end{array}$ & $\begin{array}{c}1.67 \\
(1.58)\end{array}$ & $\begin{array}{c}2.33 \\
(1.82)\end{array}$ & $\begin{array}{c}0.67 \\
(1.28)\end{array}$ & $\begin{array}{c}0.00 \\
(1.00)\end{array}$ & $\begin{array}{c}1.33 \\
(1.47)\end{array}$ \\
\hline PCH-222 & $\begin{array}{c}1.33 \\
(1.47)\end{array}$ & $\begin{array}{c}0.67 \\
(1.24)\end{array}$ & $\begin{array}{c}2.67 \\
(1.91)\end{array}$ & $\begin{array}{c}1.00 \\
(1.38)\end{array}$ & $\begin{array}{c}0.00 \\
(1.00)\end{array}$ & $\begin{array}{c}1.13 \\
\text { (1.48) }\end{array}$ \\
\hline RG-2928 & $\begin{array}{c}1.00 \\
(1.38) \\
\end{array}$ & $\begin{array}{c}2.67 \\
(1.88) \\
\end{array}$ & $\begin{array}{c}2.00 \\
(1.73)\end{array}$ & $\begin{array}{c}0.33 \\
(1.34) \\
\end{array}$ & $\begin{array}{c}0.00 \\
(1.00) \\
\end{array}$ & $\begin{array}{c}1.2 \\
(1.49) \\
\end{array}$ \\
\hline RG-1180 & $\begin{array}{c}1.67 \\
(1.58)\end{array}$ & $\begin{array}{c}1.00 \\
(1.38)\end{array}$ & $\begin{array}{c}2.67 \\
(1.88)\end{array}$ & $\begin{array}{c}0.67 \\
(1.24) \\
\end{array}$ & $\begin{array}{c}0.00 \\
(1.00)\end{array}$ & $\begin{array}{c}1.20 \\
(1.46) \\
\end{array}$ \\
\hline RG-776 & $\begin{array}{c}0.33 \\
(1.34)\end{array}$ & $\begin{array}{c}0.67 \\
(1.28)\end{array}$ & $\begin{array}{c}1.33 \\
(1.52)\end{array}$ & $\begin{array}{c}0.00 \\
(1.00)\end{array}$ & $\begin{array}{c}0.00 \\
(1.00)\end{array}$ & $\begin{array}{c}0.47 \\
(1.41) \\
\end{array}$ \\
\hline $\begin{array}{l}\text { Kranthi } \\
\text { (Check) }\end{array}$ & $\begin{array}{c}1.00 \\
(1.38)\end{array}$ & $\begin{array}{c}1.00 \\
(1.38)\end{array}$ & $\begin{array}{c}2.00 \\
(1.73)\end{array}$ & $\begin{array}{c}1.67 \\
(1.61)\end{array}$ & $\begin{array}{c}0.00 \\
(1.00)\end{array}$ & $\begin{array}{c}1.13 \\
(1.46) \\
\end{array}$ \\
\hline SEm & NS & NS & 0.16 & 0.14 & & 0.19 \\
\hline $\mathrm{C}(\mathrm{P}=0.05)$ & NS & NS & 0.33 & 0.41 & NS & 0.55 \\
\hline
\end{tabular}

Figures in parenthesis are square root transformed values.

$\mathrm{SEm}=$ Standard error of mean

$\mathrm{CD}(\mathrm{P}=0.05)=$ Critical Difference at $5 \%$ level of significance

$\mathrm{DAS}=$ Days after sowing.

NS=Non-significant 
Table.3 Effect of early sowing on incidence of tobacco caterpillar, Spodoptera litura

\begin{tabular}{|c|c|c|c|c|c|c|}
\hline \multirow[b]{2}{*}{ Genotypes } & \multicolumn{6}{|c|}{ Number of larvae/plant } \\
\hline & $\begin{array}{c}30 \text { das } \\
(30.09 .2013)\end{array}$ & $\begin{array}{c}60 \text { das } \\
(30.10 .2013)\end{array}$ & $\begin{array}{c}90 \text { das } \\
(30.11 .2013)\end{array}$ & $\begin{array}{c}120 \text { das } \\
(30.12 .2013)\end{array}$ & $\begin{array}{c}150 \text { das } \\
(30.01 .2014)\end{array}$ & Mean \\
\hline DPC-9 & $\begin{array}{c}0.00 \\
(1.00)\end{array}$ & $\begin{array}{c}0.00 \\
(1.00)\end{array}$ & $\begin{array}{c}0.00 \\
(1.00)\end{array}$ & $\begin{array}{c}0.00 \\
(1.00)\end{array}$ & $\begin{array}{c}0.00 \\
(1.00)\end{array}$ & $\begin{array}{c}0.00 \\
(1.00)\end{array}$ \\
\hline DCS-9 & $\begin{array}{c}2.00 \\
(1.72)\end{array}$ & $\begin{array}{c}2.00 \\
(1.73)\end{array}$ & $\begin{array}{c}2.00 \\
(1.72)\end{array}$ & $\begin{array}{c}4.00 \\
(2.23)\end{array}$ & $\begin{array}{c}1.00 \\
(1.38)\end{array}$ & $\begin{array}{c}2.20 \\
(1.79) \\
\end{array}$ \\
\hline $48-1$ & $\begin{array}{c}2.00 \\
(1.00) \\
\end{array}$ & $\begin{array}{c}3.00 \\
(1.79) \\
\end{array}$ & $\begin{array}{c}5.00 \\
(2.29) \\
\end{array}$ & $\begin{array}{c}4.00 \\
(2.23) \\
\end{array}$ & $\begin{array}{c}2.00 \\
(1.00) \\
\end{array}$ & $\begin{array}{r}3.20 \\
(1.89) \\
\end{array}$ \\
\hline Haritha & $\begin{array}{c}1.00 \\
(1.41) \\
\end{array}$ & $\begin{array}{c}2.00 \\
(1.73) \\
\end{array}$ & $\begin{array}{c}4.00 \\
(1.41) \\
\end{array}$ & $\begin{array}{c}2.00 \\
(1.72)\end{array}$ & $\begin{array}{c}0.00 \\
(1.00)\end{array}$ & $\begin{array}{r}1.80 \\
(1.58) \\
\end{array}$ \\
\hline PCH-288 & $\begin{array}{c}0.00 \\
(1.00) \\
\end{array}$ & $\begin{array}{c}1.00 \\
(1.38) \\
\end{array}$ & $\begin{array}{c}1.00 \\
(1.38) \\
\end{array}$ & $\begin{array}{c}4.00 \\
(2.23) \\
\end{array}$ & $\begin{array}{c}0.00 \\
(1.00) \\
\end{array}$ & $\begin{array}{r}1.20 \\
(1.43) \\
\end{array}$ \\
\hline PCH-111 & $\begin{array}{c}1.00 \\
(1.38)\end{array}$ & $\begin{array}{c}2.00 \\
(1.72)\end{array}$ & $\begin{array}{c}4.00 \\
(2.23)\end{array}$ & $\begin{array}{c}2.00 \\
(1.72)\end{array}$ & $\begin{array}{c}1.00 \\
(1.41)\end{array}$ & $\begin{array}{c}2.00 \\
(1.72)\end{array}$ \\
\hline PCH-254 & $\begin{array}{c}1.00 \\
(1.38)\end{array}$ & $\begin{array}{c}1.00 \\
(1.41)\end{array}$ & $\begin{array}{c}2.00 \\
(1.72)\end{array}$ & $\begin{array}{c}1.00 \\
(1.33)\end{array}$ & $\begin{array}{c}0.00 \\
(1.00)\end{array}$ & $\begin{array}{c}1.00 \\
(1.38)\end{array}$ \\
\hline M-574 & $\begin{array}{c}0.00 \\
(1.00)\end{array}$ & $\begin{array}{c}2.00 \\
(1.73)\end{array}$ & $\begin{array}{c}1.00 \\
(1.41)\end{array}$ & $\begin{array}{c}1.00 \\
(1.41)\end{array}$ & $\begin{array}{c}1.00 \\
(1.38)\end{array}$ & $\begin{array}{c}1.00 \\
(1.40)\end{array}$ \\
\hline Kiran & $\begin{array}{c}1.00 \\
(1.38)\end{array}$ & $\begin{array}{c}2.00 \\
(1.73)\end{array}$ & $\begin{array}{c}2.00 \\
(1.71)\end{array}$ & $\begin{array}{c}1.00 \\
(1.38)\end{array}$ & $\begin{array}{c}0.00 \\
(1.00)\end{array}$ & $\begin{array}{c}1.20 \\
(1.39)\end{array}$ \\
\hline PCH-248 & $\begin{array}{c}0.00 \\
(1.00) \\
\end{array}$ & $\begin{array}{c}1.00 \\
(1.41)\end{array}$ & $\begin{array}{c}1.00 \\
(1.41) \\
\end{array}$ & $\begin{array}{c}2.00 \\
(1.72) \\
\end{array}$ & $\begin{array}{c}1.00 \\
(1.38) \\
\end{array}$ & $\begin{array}{c}1.00 \\
(1.38) \\
\end{array}$ \\
\hline PCH-294 & $\begin{array}{c}2.00 \\
(1.73) \\
\end{array}$ & $\begin{array}{c}1.00 \\
(1.38)\end{array}$ & $\begin{array}{c}2.00 \\
(1.72) \\
\end{array}$ & $\begin{array}{c}3.00 \\
(1.79) \\
\end{array}$ & $\begin{array}{c}0.00 \\
(1.00) \\
\end{array}$ & $\begin{array}{r}1.60 \\
(1.75) \\
\end{array}$ \\
\hline PCH-262 & $\begin{array}{c}1.00 \\
(1.38)\end{array}$ & $\begin{array}{c}3.00 \\
(1.79)\end{array}$ & $\begin{array}{c}6.00 \\
(1.00)\end{array}$ & $\begin{array}{c}1.00 \\
(1.41)\end{array}$ & $\begin{array}{c}0.00 \\
(1.00)\end{array}$ & $\begin{array}{c}2.20 \\
(1.08)\end{array}$ \\
\hline GCH-4 & $\begin{array}{c}0.00 \\
(1.00)\end{array}$ & $\begin{array}{c}1.00 \\
(1.41)\end{array}$ & $\begin{array}{c}2.00 \\
(1.73)\end{array}$ & $\begin{array}{c}2.00 \\
(1.73)\end{array}$ & $\begin{array}{c}1.00 \\
(1.38)\end{array}$ & $\begin{array}{c}1.20 \\
(1.46)\end{array}$ \\
\hline PCH-282 & $\begin{array}{c}2.00 \\
(1.72)\end{array}$ & $\begin{array}{c}1.00 \\
(1.73)\end{array}$ & $\begin{array}{c}2.00 \\
(1.72)\end{array}$ & $\begin{array}{c}1.00 \\
(1.41)\end{array}$ & $\begin{array}{c}0.00 \\
(1.00)\end{array}$ & $\begin{array}{c}1.20 \\
(1.46)\end{array}$ \\
\hline RG-2835 & $\begin{array}{c}2.00 \\
(1.72)\end{array}$ & $\begin{array}{c}3.00 \\
(1.72)\end{array}$ & $\begin{array}{c}4.00 \\
(2.23)\end{array}$ & $\begin{array}{c}2.00 \\
(1.00)\end{array}$ & $\begin{array}{c}1.00 \\
(1.38)\end{array}$ & $\begin{array}{r}2.40 \\
(1.23)\end{array}$ \\
\hline PCH-106 & $\begin{array}{c}1.00 \\
(1.41)\end{array}$ & $\begin{array}{c}2.00 \\
(1.73)\end{array}$ & $\begin{array}{c}3.00 \\
(1.79)\end{array}$ & $\begin{array}{c}1.00 \\
(1.41)\end{array}$ & $\begin{array}{c}0.00 \\
(1.00)\end{array}$ & $\begin{array}{r}1.40 \\
(1.63)\end{array}$ \\
\hline PCH-222 & $\begin{array}{c}0.00 \\
(1.00)\end{array}$ & $\begin{array}{c}2.00 \\
(1.66)\end{array}$ & $\begin{array}{c}4.00 \\
(2.23) \\
\end{array}$ & $\begin{array}{c}1.00 \\
(1.37) \\
\end{array}$ & $\begin{array}{c}0.00 \\
(1.00)\end{array}$ & $\begin{array}{r}1.40 \\
(1.23) \\
\end{array}$ \\
\hline RG-2928 & $\begin{array}{c}0.00 \\
(1.00)\end{array}$ & $\begin{array}{c}3.00 \\
(1.99)\end{array}$ & $\begin{array}{c}0.00 \\
(1.00)\end{array}$ & $\begin{array}{c}2.00 \\
(1.72)\end{array}$ & $\begin{array}{c}0.00 \\
(1.00)\end{array}$ & $\begin{array}{c}1.00 \\
(1.35) \\
\end{array}$ \\
\hline RG-1180 & $\begin{array}{c}1.00 \\
(1.38)\end{array}$ & $\begin{array}{c}1.00 \\
(1.41)\end{array}$ & $\begin{array}{c}5.00 \\
(2.27)\end{array}$ & $\begin{array}{c}3.00 \\
(1.79)\end{array}$ & $\begin{array}{c}2.00 \\
(1.72)\end{array}$ & $\begin{array}{c}2.40 \\
(2.43)\end{array}$ \\
\hline RG-776 & $\begin{array}{c}1.00 \\
(1.38)\end{array}$ & $\begin{array}{c}3.00 \\
(1.79)\end{array}$ & $\begin{array}{c}2.00 \\
(1.72)\end{array}$ & $\begin{array}{c}2.00 \\
(1.72)\end{array}$ & $\begin{array}{c}0.00 \\
(1.00)\end{array}$ & $\begin{array}{c}1.60 \\
(1.23) \\
\end{array}$ \\
\hline $\begin{array}{l}\text { Kranthi } \\
\text { (Check) }\end{array}$ & $\begin{array}{c}2.00 \\
(1.72)\end{array}$ & $\begin{array}{c}1.00 \\
(1.38)\end{array}$ & $\begin{array}{c}5.00 \\
(2.27)\end{array}$ & $\begin{array}{c}2.00 \\
(1.72)\end{array}$ & $\begin{array}{c}2.00 \\
(2.23)\end{array}$ & $\begin{array}{c}2.40 \\
(2.41)\end{array}$ \\
\hline SEm & 0.09 & 0.13 & 0.10 & 0.13 & 0.10 & 0.13 \\
\hline $\mathrm{CD}(\mathrm{P}=0.05)$ & 0.25 & 0.37 & 0.30 & 0.36 & 0.29 & 0.33 \\
\hline
\end{tabular}

Figures in parenthesis are square root transformed values.

$\mathrm{SEm}=$ Standard error of mean

$\mathrm{CD}(\mathrm{P}=0.05)=$ Critical Difference at $5 \%$ level of significance

$\mathrm{DAS}=$ Days after sowing. 
Table.4 Effect of late sowing on incidence of tobacco caterpillar, Spodoptera litura

\begin{tabular}{|c|c|c|c|c|c|c|}
\hline \multirow[b]{2}{*}{ Genotypes } & \multicolumn{6}{|c|}{ Number of larvae/plant } \\
\hline & $\begin{array}{c}30 \text { das } \\
(30.09 .2013)\end{array}$ & $\begin{array}{c}60 \text { das } \\
(30.10 .2013)\end{array}$ & $\begin{array}{c}90 \text { das } \\
(30.11 .2013)\end{array}$ & $\begin{array}{c}120 \text { das } \\
(30.12 .2013)\end{array}$ & $\begin{array}{c}150 \text { das } \\
(30.01 .2014)\end{array}$ & Mean \\
\hline DPC-9 & $\begin{array}{c}0.00 \\
(1.00) \\
\end{array}$ & $\begin{array}{c}0.00 \\
(1.00) \\
\end{array}$ & $\begin{array}{c}0.00 \\
(1.00) \\
\end{array}$ & $\begin{array}{c}0.00 \\
(1.00) \\
\end{array}$ & $\begin{array}{c}0.00 \\
(1.00) \\
\end{array}$ & $\begin{array}{c}0.00 \\
(1.00)\end{array}$ \\
\hline DCS-9 & $\begin{array}{c}2.00 \\
(1.73) \\
\end{array}$ & $\begin{array}{c}3.00 \\
(2.00) \\
\end{array}$ & $\begin{array}{c}0.00 \\
(1.00) \\
\end{array}$ & $\begin{array}{c}2.00 \\
(1.72)\end{array}$ & $\begin{array}{c}0.00 \\
(1.00)\end{array}$ & $\begin{array}{c}1.40 \\
(1.49)\end{array}$ \\
\hline $48-1$ & $\begin{array}{c}1.00 \\
(1.38)\end{array}$ & $\begin{array}{c}0.00 \\
(1.00)\end{array}$ & $\begin{array}{c}0.00 \\
(1.00)\end{array}$ & $\begin{array}{c}0.00 \\
(1.00)\end{array}$ & $\begin{array}{c}0.00 \\
(1.00)\end{array}$ & $\begin{array}{c}0.20 \\
(1.08)\end{array}$ \\
\hline Haritha & $\begin{array}{c}1.00 \\
(1.41) \\
\end{array}$ & $\begin{array}{c}0.00 \\
(1.00) \\
\end{array}$ & $\begin{array}{c}2.00 \\
(1.72) \\
\end{array}$ & $\begin{array}{c}1.00 \\
(1.41) \\
\end{array}$ & $\begin{array}{c}0.00 \\
(1.00) \\
\end{array}$ & $\begin{array}{c}0.80 \\
(1.31) \\
\end{array}$ \\
\hline PCH-288 & $\begin{array}{c}1.00 \\
(1.41) \\
\end{array}$ & $\begin{array}{c}1.00 \\
(1.41) \\
\end{array}$ & $\begin{array}{c}2.00 \\
(1.73) \\
\end{array}$ & $\begin{array}{c}2.00 \\
(1.73) \\
\end{array}$ & $\begin{array}{c}0.00 \\
(1.00) \\
\end{array}$ & $\begin{array}{c}1.20 \\
(1.46) \\
\end{array}$ \\
\hline PCH-111 & $\begin{array}{c}0.00 \\
(1.00)\end{array}$ & $\begin{array}{c}0.00 \\
(1.00)\end{array}$ & $\begin{array}{c}2.00 \\
(1.72)\end{array}$ & $\begin{array}{c}2.00 \\
(1.72)\end{array}$ & $\begin{array}{c}0.00 \\
(1.00)\end{array}$ & $\begin{array}{c}0.80 \\
(1.29)\end{array}$ \\
\hline $\mathrm{PCH}-254$ & $\begin{array}{c}1.00 \\
(1.38) \\
\end{array}$ & $\begin{array}{c}1.00 \\
(1.38)\end{array}$ & $\begin{array}{c}2.00 \\
(1.73) \\
\end{array}$ & $\begin{array}{c}1.67 \\
(1.63) \\
\end{array}$ & $\begin{array}{c}0.00 \\
(1.00)\end{array}$ & $\begin{array}{c}1.13 \\
(1.44) \\
\end{array}$ \\
\hline M-574 & $\begin{array}{c}1.00 \\
(1.38) \\
\end{array}$ & $\begin{array}{c}1.00 \\
(1.41)\end{array}$ & $\begin{array}{c}0.00 \\
(1.00) \\
\end{array}$ & $\begin{array}{c}1.00 \\
(1.38)\end{array}$ & $\begin{array}{c}0.00 \\
(1.00) \\
\end{array}$ & $\begin{array}{c}0.60 \\
(1.25) \\
\end{array}$ \\
\hline Kiran & $\begin{array}{c}0.00 \\
(1.00) \\
\end{array}$ & $\begin{array}{c}1.00 \\
(1.41) \\
\end{array}$ & $\begin{array}{c}0.00 \\
(1.00) \\
\end{array}$ & $\begin{array}{c}0.00 \\
(1.00) \\
\end{array}$ & $\begin{array}{c}0.00 \\
(1.00) \\
\end{array}$ & $\begin{array}{c}0.20 \\
(1.08) \\
\end{array}$ \\
\hline PCH-248 & $\begin{array}{c}1.00 \\
(1.41)\end{array}$ & $\begin{array}{c}0.00 \\
(1.00)\end{array}$ & $\begin{array}{c}0.00 \\
(1.00)\end{array}$ & $\begin{array}{c}1.00 \\
(1.41)\end{array}$ & $\begin{array}{c}0.00 \\
(1.00)\end{array}$ & $\begin{array}{c}0.40 \\
(1.17)\end{array}$ \\
\hline PCH-294 & $\begin{array}{c}0.00 \\
(1.00)\end{array}$ & $\begin{array}{c}1.00 \\
(1.38)\end{array}$ & $\begin{array}{c}2.00 \\
(1.73)\end{array}$ & $\begin{array}{c}0.00 \\
(1.00)\end{array}$ & $\begin{array}{c}0.00 \\
(1.00)\end{array}$ & $\begin{array}{c}0.60 \\
(1.23)\end{array}$ \\
\hline PCH-262 & $\begin{array}{c}0.00 \\
(1.00)\end{array}$ & $\begin{array}{c}0.00 \\
(1.00)\end{array}$ & $\begin{array}{c}0.00 \\
(1.00)\end{array}$ & $\begin{array}{c}1.33 \\
(1.52) \\
\end{array}$ & $\begin{array}{c}0.00 \\
(1.00) \\
\end{array}$ & $\begin{array}{c}0.27 \\
(1.11)\end{array}$ \\
\hline GCH-4 & $\begin{array}{c}1.00 \\
(1.41)\end{array}$ & $\begin{array}{c}1.00 \\
(1.38)\end{array}$ & $\begin{array}{c}2.00 \\
(1.72) \\
\end{array}$ & $\begin{array}{c}0.00 \\
(1.00)\end{array}$ & $\begin{array}{c}0.00 \\
(1.00)\end{array}$ & $\begin{array}{c}0.80 \\
(1.31) \\
\end{array}$ \\
\hline PCH-282 & $\begin{array}{c}2.00 \\
(1.73)\end{array}$ & $\begin{array}{c}1.00 \\
(1.41)\end{array}$ & $\begin{array}{c}1.00 \\
(1.41)\end{array}$ & $\begin{array}{c}0.00 \\
(1.00)\end{array}$ & $\begin{array}{c}0.00 \\
(1.00)\end{array}$ & $\begin{array}{c}0.80 \\
(1.31) \\
\end{array}$ \\
\hline RG-2835 & $\begin{array}{c}1.00 \\
(1.41) \\
\end{array}$ & $\begin{array}{c}0.00 \\
(1.00) \\
\end{array}$ & $\begin{array}{c}0.00 \\
(1.00) \\
\end{array}$ & $\begin{array}{c}0.00 \\
(1.00) \\
\end{array}$ & $\begin{array}{c}0.00 \\
(1.00) \\
\end{array}$ & $\begin{array}{c}0.20 \\
(1.08) \\
\end{array}$ \\
\hline PCH-106 & $\begin{array}{c}1.00 \\
(1.38) \\
\end{array}$ & $\begin{array}{c}0.00 \\
(1.00)\end{array}$ & $\begin{array}{c}0.00 \\
(1.00)\end{array}$ & $\begin{array}{c}1.00 \\
(1.41) \\
\end{array}$ & $\begin{array}{c}0.00 \\
(1.00) \\
\end{array}$ & $\begin{array}{c}0.40 \\
(1.17)\end{array}$ \\
\hline PCH-222 & $\begin{array}{c}2.00 \\
(1.72)\end{array}$ & $\begin{array}{c}2.00 \\
(1.72)\end{array}$ & $\begin{array}{c}2.00 \\
(1.73)\end{array}$ & $\begin{array}{c}0.00 \\
(1.00)\end{array}$ & $\begin{array}{c}0.00 \\
(1.00)\end{array}$ & $\begin{array}{c}1.20 \\
(1.44)\end{array}$ \\
\hline RG-2928 & $\begin{array}{c}0.00 \\
(1.00)\end{array}$ & $\begin{array}{c}0.00 \\
(1.00)\end{array}$ & $\begin{array}{c}1.00 \\
(1.38)\end{array}$ & $\begin{array}{c}0.00 \\
(1.00)\end{array}$ & $\begin{array}{c}0.00 \\
(1.00)\end{array}$ & $\begin{array}{c}0.20 \\
(1.08)\end{array}$ \\
\hline RG-1180 & $\begin{array}{c}0.00 \\
(1.00) \\
\end{array}$ & $\begin{array}{c}2.00 \\
(1.73) \\
\end{array}$ & $\begin{array}{c}0.00 \\
(1.00) \\
\end{array}$ & $\begin{array}{c}2.00 \\
(1.72)\end{array}$ & $\begin{array}{c}0.00 \\
(1.00) \\
\end{array}$ & $\begin{array}{c}0.80 \\
(1.29) \\
\end{array}$ \\
\hline RG-776 & $\begin{array}{c}2.00 \\
(1.73) \\
\end{array}$ & $\begin{array}{c}0.00 \\
(1.00) \\
\end{array}$ & $\begin{array}{c}1.00 \\
(1.38) \\
\end{array}$ & $\begin{array}{c}0.00 \\
(1.00) \\
\end{array}$ & $\begin{array}{c}0.00 \\
(1.00) \\
\end{array}$ & $\begin{array}{c}0.60 \\
(1.23) \\
\end{array}$ \\
\hline Kranthi & $\begin{array}{c}1.00 \\
(1.41)\end{array}$ & $\begin{array}{c}0.00 \\
(1.00)\end{array}$ & $\begin{array}{c}1.00 \\
(1.41)\end{array}$ & $\begin{array}{c}0.00 \\
(1.00)\end{array}$ & $\begin{array}{c}0.00 \\
(1.00)\end{array}$ & $\begin{array}{c}0.40 \\
(1.17)\end{array}$ \\
\hline SEm & 0.10 & 0.08 & 0.09 & 0.09 & & 0.12 \\
\hline $\mathrm{CD}(\mathrm{P}=0.05)$ & 0.28 & 0.23 & 0.27 & 0.25 & NS & 0.29 \\
\hline
\end{tabular}

Figures in parenthesis are square root transformed values.

$\mathrm{SEm}=$ Standard error of mean

$\mathrm{CD}(\mathrm{P}=0.05)=$ Critical Difference at $5 \%$ level of significance

$\mathrm{DAS}=$ Days after sowing.

At 90 days age of crop, maximum of 2.00 larvae per plant was observed in Haritha, $\mathrm{PCH}-$
288, PCH-111, PCH-254, PCH-294, GCH-4 and PCH-222 which were on par with each 
other, followed by PCH-282, RG-2928, RG-776 and Kranthi with one (1.00) larva per plant and no infestation of $S$. litura was recorded in genotypes DPC-9, DCS-9, 48-1, M-574, Kiran, PCH-248, PCH-262, RG-2835, PCH-106 and RG-1180. At 120 days age of the crop, maximum of 2.00 larvae was recorded on DCS9, PCH-288, PCH-111 and RG-1180. The genotype, $\mathrm{PCH}-254$ has recorded 1.67 larvae per plant followed by 1.33 larvae in $\mathrm{PCH}-262$. Minimum of 1.00 larva was observed on the genotypes, Haritha, M-574, $\mathrm{PCH}-248$ and $\mathrm{PCH}-$ 106. No infestation of $S$. litura was observed on genotypes DPC-9, 48-1, Kiran, PCH-294, GCH-4, PCH-282, RG-2835, PCH-222, RG2928, RG-1180, RG-776 and Kranthi. At 150 days after sowing no incidence of $S$. litura was observed in all the genotypes. On an average, no $S$. litura incidence was observed on DPC-9 throughout the crop growth period and maximum of 1.40 larvae was recorded on DCS9. However, mean number of larvae of 1.20 per plant was observed on $\mathrm{PCH}-288$ and $\mathrm{PCH}-222$ followed by 1.13 larvae per plant on $\mathrm{PCH}-262$; 0.80 larvae per plant were recorded on genotypes, Haritha, PCH-111, GCH-4, PCH282 and RG- 1180. Larval population of 0.60 was recorded on M-574, PCH-294 and RG-776, followed by 0.40 larvae per plant on the genotypes, $\mathrm{PCH}-248, \mathrm{PCH}-106$ and Kranti; 0.27 larvae per plant were recorded on $\mathrm{PCH}-262$ and minimum of 0.20 larvae was recorded on $48-1$, Kiran, RG-2835 and RG-2928.

It is clear from the study that occurrence of both A. janata and $S$ litura was comparatively more in early sown crop than late sown crop. Similar results reported by (APR castor, 1986). However, in some genotypes, there was a slight increase in the population of $A$. janata i.e. in the case of DCS-9 and PCH-248 when the crop was raised late by one month during August.
Similarly, with respect to $S$. litura, the genotypes $\mathrm{PCH}-254$ has recorded slight increase in the number of larvae. Present results confirm the observations made by Jayaraj and Basheer (1964), Jayraj (1966) and (Akashe et al., 2015).

\section{References}

Akashe, V. B., Indi, D. V., Patil, S. R., Jadhav, J. D. and Pawar, P. B. (2015). Incidence of insect pest damage in castor in relation to meteorological parameters in the scarcity zone of Maharashtra. Journal of Agrometeorology 17 (1): 139-141.

Annual progress report on castor. (1986). Annual castor research workers group meeting at directorate of oilseeds research, hyderabad-500 030.

DOR (2005). Integrated Pest Management in Oilseed Crops. 2nd revised edition, Directorate of Oilseeds Research, Hyderabad.

Jayaraj, S and Basheer, M. (1964). Biological observations on the castor leaf - hopper, Empoasca flavescens (F) (Jassidae: Homoptera). Madras Agricultural Journal. 51: 89-90.

Jayaraj, S. (1966). Influence of sowing times of castor varieties on their resistance to the leafhopper, Empoasca flavescens (homoptera, jassidae). Entomological experimental application. 9: 359-369.

Lakshminarayana, M. and Raoof, M. A. (2005). Insect pests and diseases of castor and their management. Directorate of Oilseeds Research. 78.

Sarma, A. K., Singh, M. P and Singh, K. I. (2005). Studies on insect-pests of castor in the agro-ecosystem of Manipur. Journal of Applied Zoological Researches. 16(2): 164-165.

\section{How to cite this article:}

Laxman, G. and Uma Maheswari, T. 2017. Effect of Dates of Sowing on Incidence of Insect Pests on Castor Genotypes. Int.J.Curr.Microbiol.App.Sci. 6(11): 1268-1275. doi: https://doi.org/10.20546/ijcmas.2017.611.151 